PROCEEDINGS OF THE

AMERICAN MATHEMATICAL SOCIETY

Volume 135, Number 7, July 2007, Pages 2095-2105

S 0002-9939(07)08705-9

Article electronically published on February 2, 2007

\title{
SOME RESULTS ON THE HOCHSCHILD COHOMOLOGY OF GROUP ALGEBRAS
}

\author{
A. POURABBAS
}

(Communicated by Joseph A. Ball)

\begin{abstract}
In this paper we investigate structure of the second cohomology of a discrete group $G$. First, for a $G$-set $S$ we show that an isomorphism of vector spaces from $\mathcal{H}^{n}\left(\ell^{1}(G), \ell^{\infty}(S)\right)$ onto $\bigoplus_{i \in I}^{\infty} \mathcal{H}^{n}\left(\ell^{1}(G), \ell^{\infty}\left(\mathcal{C}_{i}\right)\right)$ exists, where $\left\{\mathcal{C}_{i}\right\}_{i \in I}$ is the set of orbits of $S$. Next we define the notion of pseudoderivation and apply it for the calculation of $\mathcal{H}_{b, 2}^{2}\left(\ell^{1}(G), \ell^{\infty}(S)\right)$.
\end{abstract}

\section{INTRODUCTION}

In this paper we investigate structure of the second bounded cohomology of a discrete group $G$ with coefficients in $\ell^{\infty}(G)$. The theory of bounded cohomology of discrete groups is a part of the theory of the cohomology of Banach algebras which was developed by Johnson [6]. We also refer the reader to the book by Helemskii [4]. Johnson [6] used this theory to show that $\mathcal{H}^{2}\left(\ell^{1}(G), \mathbb{C}\right) \neq 0$ and so $\mathcal{H}^{2}\left(\ell^{1}(G), \ell^{1}(G)\right) \neq 0$, where $G$ is the free group on two generators. Brooks [1] showed that for a large class of discrete groups $\mathcal{H}_{b}^{2}(G) \cong \mathcal{H}^{2}\left(\ell^{1}(G), \mathbb{C}\right) \neq 0$.

In [5] Ivanov and in 7] Matsumoto and Morita showed that $\mathcal{H}^{2}\left(\ell^{1}(G), \mathbb{C}\right)$ is a Banach space for every discrete group $G$ with trivial action on $\mathbb{C}$. In $[8$ the author showed that $\mathcal{H}^{2}\left(\ell^{1}(G), \ell^{\infty}(S)\right)$ is a Banach space for every $G$-set $S$ and in general for every locally compact group $G$ he showed that $\mathcal{H}^{2}\left(L^{1}(G), L^{\infty}(G)\right)$ is a Banach space. Grigorchuk [2] developed a method based on using the space of pseudocharacters and applied it to calculate $\mathcal{H}_{b}^{2}(G)$, where $G$ is either one of a free group $\mathbb{F}_{m}$ of finite or countable rank $m$, a torus knot group or a surface group. Finally it is well known that $\mathcal{H}_{b}^{n}(G)=0$ for all $n$ if $G$ is amenable.

In this article we want to investigate a generalization of Grigorchuk's result in the case where the coefficients are in $\ell^{\infty}\left(\mathcal{C}_{i}\right)$ instead of $\mathbb{R}$, where $\mathcal{C}_{i}$ is an orbit in $G$-set $S$.

\section{Some BAsic FACTS And SOME CONVEntions}

In this section we consider the relationship between bounded cohomology and Hochschild cohomology of groups. Let $G$ be a locally compact group. A left Banach $G$-module $X$ is a Banach space $X$ which is a left $G$-module such that

(i) there exists a constant $C>0$ with $\|g x\| \leq C\|x\|$ for all $g \in G, x \in X$, and

(ii) for any $y \in X$ the map $G \rightarrow X$ given by $g \mapsto g y$ is continuous.

Received by the editors February 23, 2005 and, in revised form, March 9, 2006. 2000 Mathematics Subject Classification. Primary 43A20; Secondary 46M20. 
Similarly we define the notions of a right Banach $G$-module and a two-sided Banach $G$-module. Throughout, a Banach $G$-module means a two-sided Banach $G$-module. If $X$ is a Banach $G$-module, then the dual space $X^{\prime}$ becomes a $G$-module by defining

$$
(g \phi)(x)=\phi(x g) \quad \text { and } \quad(\phi g)(x)=\phi(g x) \quad\left(x \in X, \phi \in X^{\prime}, g \in G\right) .
$$

The dual space $X^{\prime}$ is a Banach $G$-module except that the maps $g \mapsto g y$ and $g \mapsto y g$ are possibly continuous only in the weak*-topology on $X^{\prime}$. The dual space $X^{\prime}$ will be called a dual $G$-module. If $G$ is discrete, then (ii) is trivially satisfied so that if $X$ is a Banach $G$-module, then $X^{\prime}$ is a Banach $G$-module.

Let $G$ be a locally compact group and let $X$ be a $G$-module. Let $C^{n}(G, X)$ be the space of all functions from $G \times \cdots \times G$ to $X$ and let $C^{0}(G, X)=X$. The linear mappings $\delta^{n}: C^{n-1}(G, X) \rightarrow C^{n}(G, X)$ are given by

$$
\begin{aligned}
\delta^{n} \phi\left(g_{1}, \ldots, g_{n}\right)= & g_{1} \phi\left(g_{2}, \ldots, g_{n}\right) \\
& +\sum_{j=1}^{n-1}(-1)^{j} \phi\left(g_{1}, \ldots, g_{j} g_{j+1}, \ldots, g_{n}\right) \\
& +(-1)^{n} \phi\left(g_{1}, \ldots, g_{n-1}\right) g_{n},
\end{aligned}
$$

where $\phi \in C^{n-1}(G, X)$ and $g_{1}, \ldots, g_{n} \in G$. It is easy to see that $\delta^{n+1} \delta^{n}=0$ for every $n \in \mathbb{N}=\{1,2, \ldots\}$. The cohomology of $G$ with coefficients in $X$ is

$$
H^{n}(G, X)=\operatorname{ker} \delta^{n+1} / \operatorname{Im} \delta^{n} .
$$

Now let $X$ be a Banach $G$-module. Then the Banach space $C_{b}^{n}(G, X)$ is the space of all bounded functions from $G \times \cdots \times G$ to $X$ with the $\|\cdot\|_{\infty}$-norm. The restriction of the above map $\delta^{n}$ defines a map $\delta_{b}^{n}: C_{b}^{n-1}(G, X) \rightarrow C_{b}^{n}(G, X)$ for $n \in \mathbb{N}$. The bounded cohomology of $G$ with coefficients in $X$ is

$$
\mathcal{H}_{b}^{n}(G, X)=\operatorname{ker} \delta_{b}^{n+1} / \operatorname{Im} \delta_{b}^{n} .
$$

Actually $\mathcal{H}_{b}^{n}(G, X)$ is isomorphic to $\mathcal{H}^{n}\left(L^{1}(G), X\right)$, the Hochschild cohomology of $L^{1}(G)$ with coefficients in $X$, because there is a one-to-one correspondence between Banach $G$-modules and Banach $L^{1}(G)$-modules, and

$$
\begin{aligned}
C_{b}^{n}(G, X) & =\ell^{\infty}(G \times \cdots \times G, X) \\
& =\mathcal{L}^{n}\left(L^{1}(G), X\right),
\end{aligned}
$$

where $\mathcal{L}^{n}\left(\ell^{1}(G), X\right)$ is the space of bounded $n$-linear maps from $\ell^{1}(G) \times \cdots \times \ell^{1}(G)$ (n-times) into $X$.

Let $X$ be a Banach $G$-module. We define a new $G$-module structure on $X$ by

$$
g \circ x=x, \quad x \circ g=g^{-1} x g
$$

and denote the resulting module by $X^{\circ}$. Then the isomorphism

$$
\mathcal{H}^{n}\left(L^{1}(G), X^{\prime}\right) \cong \mathcal{H}^{n}\left(L^{1}(G),\left(X^{\circ}\right)^{\prime}\right)
$$

holds [6]. Thus in cohomology theory it is enough to consider Banach $G$-modules with trivial action on one side.

If $X=\mathbb{C}$ we write $\mathcal{H}_{b}^{n}(G)$ instead of $\mathcal{H}_{b}^{n}(G, \mathbb{C})$. It is well known that $\mathcal{H}_{b}^{1}(G)=0$ for all discrete groups $G$, because if $\phi \in C_{b}^{1}(G)$ such that $\delta \phi=0$, then

$$
\delta \phi\left(g_{1}, g_{2}\right)=\phi\left(g_{1}\right)-\phi\left(g_{1} g_{2}\right)+\phi\left(g_{2}\right)=0 .
$$

By induction $\phi\left(g^{n}\right)=n \phi(g)$ for every $n$. Therefore $\phi=0$, that is, there do not exist nontrivial bounded homomorphisms from $G$ into $\mathbb{C}$. 
The space $C_{b}^{n}(G)$ with the norm $\|\cdot\|_{\infty}$ is a Banach space. Therefore in $\mathcal{H}_{b}^{n}(G)$ there is a seminorm $\|g\|=\inf _{f \in[g]}\|f\|_{\infty}$, where $[g]$ denotes the cohomology class corresponding to $g \in C_{b}^{n}(G)$. This seminorm is a norm if and only if $\operatorname{Im} \delta_{b}^{n}$ is a closed subspace of $C_{b}^{n}(G)$, which means $\mathcal{H}_{b}^{n}(G)$ is a Banach space. It is unknown whether or not $\mathcal{H}_{b}^{n}(G)$ is a Banach space for every $n \geq 3$ and arbitrary locally compact group $G$. Meanwhile for the free group on two generators $G=\mathbb{F}_{2}$ and any discrete group $G$ admitting a surjective homomorphism $f: G \rightarrow \mathbb{F}_{2}$, Soma [9] showed that $\mathcal{H}_{b}^{3}(G)$ is not a Banach space. He also showed that there exists a finitely generated discrete group $G$ such that $\mathcal{H}_{b}^{n}(G)$ is not a Banach space for any $n \geq 5$.

Let $G$ be a discrete group, and let $S$ be a $G$-set with trivial action on the left-hand side. We define the relation $\sim$ on $S$ by setting $x_{1} \sim x_{2}$ if and only if $x_{1}, x_{2} \in S$, and there is an element $g \in G$ such that $x_{1} g=x_{2}$. The relation $\sim$ is an equivalence relation on $S$. So $S$ is partitioned into disjoint equivalence classes with respect to the equivalence relation $\sim$. These equivalence classes are called the orbits of the action. For each $x \in S$, the equivalence class containing $x$ is called the orbit of $x$, which is the subset $\{x g: g \in G\}$ of $S$.

The set $\operatorname{Stab}_{G}(x)=\{g \in G: x g=x\}$, which is a subgroup of $G$, is called the stabiliser of $x$ in $G$. If $H$ is a subgroup of $G$, a right transversal of $H$ in $G$ is a subset $T$ of $G$ consisting of precisely one element from each right coset of $H$ in $G$. Observe that $H T=G$ and $|T|=|G: H|$, where $|T|$ denotes the number of elements in $T$ and $|G: H|$ is the index of $H$ in $G$. Throughout by a transversal of $H$ in $G$ we mean a right transversal of $H$ in $G$. Let $\left\{\mathcal{C}_{i}\right\}_{i \in I}$ be the set of orbits of $S$, which is a partition for $S$. Let $\mathcal{L}^{n}\left(\ell^{1}(G), \ell^{\infty}\left(\mathcal{C}_{i}\right)\right)$ be the space of bounded $n$-linear functions from $\ell^{1}(G) \times \cdots \times \ell^{1}(G)\left(n\right.$-times) into $\ell^{\infty}\left(\mathcal{C}_{i}\right)$. (We identify $\mathcal{L}^{0}\left(\ell^{1}(G), \ell^{\infty}\left(\mathcal{C}_{i}\right)\right)$ with $\ell^{\infty}\left(\mathcal{C}_{i}\right)$.) For each $n \in \mathbb{N}$ and each $i \in I$ the boundary map

$$
\delta_{i}^{n}: \mathcal{L}^{n-1}\left(\ell^{1}(G), \ell^{\infty}\left(\mathcal{C}_{i}\right)\right) \rightarrow \mathcal{L}^{n}\left(\ell^{1}(G), \ell^{\infty}\left(\mathcal{C}_{i}\right)\right)
$$

is defined by

$$
\begin{aligned}
\delta_{i}^{n} \phi_{i}\left(g_{1}, \ldots, g_{n}\right)[c] & =\phi_{i}\left(g_{2}, \ldots, g_{n}\right)\left[c g_{1}\right] \\
& +\sum_{j=1}^{n-1}(-1)^{j} \phi_{i}\left(g_{1}, \ldots, g_{j} g_{j+1}, \ldots, g_{n}\right)[c] \\
& +(-1)^{n} \phi_{i}\left(g_{1}, \ldots, g_{n-1}\right)[c]
\end{aligned}
$$

where $\phi_{i} \in \mathcal{L}^{n-1}\left(\ell^{1}(G), \ell^{\infty}\left(\mathcal{C}_{i}\right)\right), g_{1}, \ldots, g_{n} \in G$ and $c \in \mathcal{C}_{i}$. We have $\delta_{i}^{n+1} \delta_{i}^{n}=0$, and we put $\mathcal{H}^{n}\left(\ell^{1}(G), \ell^{\infty}\left(\mathcal{C}_{i}\right)\right)=\operatorname{ker} \delta_{i}^{n+1} / \operatorname{Im} \delta_{i}^{n}$ which is the cohomology group of $\ell^{1}(G)$ with coefficients in $\ell^{\infty}\left(\mathcal{C}_{i}\right)$. We will often drop the superscripts on $\delta_{i}^{n}$.

\section{The COHOMOlOGY OF DISCRETE GROUPS}

Let $G$ be a discrete group, and let $S$ be a $G$-set with trivial action on the left-hand side.

Lemma 3.1. If $\mathcal{C}_{i}$ is an orbit in $S, c \in \mathcal{C}_{i}$ and if $T$ is a transversal of $\operatorname{Stab}_{G}(c)$ in $G$, then

(i) $\mathcal{C}_{i}=\{c t\}_{t \in T}$, and for every $s \in \mathcal{C}_{i}$ there is a unique $t \in T$ such that $s=c t$.

(ii) For every $g$ in $G, \operatorname{Stab}_{G}(c g)=\operatorname{Stab}_{G}(c)^{g}$, where $\operatorname{Stab}_{G}(c)^{g}$ is equal to $\left\{g^{-1} x g: x \in \operatorname{Stab}_{G}(c)\right\}$.

(iii) For every $g \in G, g^{-1} T$ is a transversal for $\operatorname{Stab}_{G}(c g)$. 
Proof. (i) It is obvious that $\{c t\}_{t \in T} \subseteq \mathcal{C}_{i}$. Now if $k \in \mathcal{C}_{i}$, then there exists a $g \in G$ such that $k=c g$ but $g=h t$ for unique elements $h \in \operatorname{Stab}_{G}(c)$ and $t \in T$, so $k=c h t=c t$ and so $k \in\{c t\}_{t \in T}$. Now if $t_{1}, t_{2} \in T$ such that $c t_{1}=c t_{2}$, then $c t_{1} t_{2}^{-1}=c$, that means $t_{1} t_{2}^{-1} \in \operatorname{Stab}_{G}(c)$, therefore $t_{1}=t_{2}$.

(ii) Let $h \in \operatorname{Stab}_{G}(c)$. Since $(c g)\left(g^{-1} h g\right)=c h g=c g$, then $g^{-1} h g \in \operatorname{Stab}_{G}(c g)$, that is, $\operatorname{Stab}_{G}(c)^{g} \subseteq \operatorname{Stab}_{G}(c g)$. Now let $k \in \operatorname{Stab}_{G}(c g)$. Since $c\left(g k g^{-1}\right)=$ $(c g k) g^{-1}=(c g) g^{-1}=c$, then $g k g^{-1} \in \operatorname{Stab}_{G}(c)$, that is, $k \in g^{-1} \operatorname{Stab}_{G}(c) g$, therefore $\operatorname{Stab}_{G}(c g) \subseteq \operatorname{Stab}_{G}(c)^{g}$.

(iii) If we consider any right coset $\operatorname{Stab}_{G}(c) x$, then by hypothesis $\left|T \cap \operatorname{Stab}_{G}(c) x\right|$ $=1$, and so $\left|g^{-1} T \cap g^{-1} \operatorname{Stab}_{G}(c) g g^{-1} x\right|=\left|g^{-1} T \cap \operatorname{Stab}_{G}(c g) x^{\prime}\right|=1$, where $x^{\prime}=$ $g^{-1} x$.

Lemma 3.2. Let $\psi_{i} \in \mathcal{L}^{1}\left(\ell^{1}(G), \ell^{\infty}\left(\mathcal{C}_{i}\right)\right)$. Then for every $c \in \mathcal{C}_{i}$

(i) $\left|\psi_{i}(g)[c]\right| \leq\left\|\delta_{i} \psi_{i}\right\|$ for all $g$ in $\operatorname{Stab}_{G}(c)$.

(ii) $\left|\psi_{i}(h)[c]-\psi_{i}(g h)[c]\right| \leq 2\left\|\delta_{i} \psi_{i}\right\|$ for all $g$ in $\operatorname{Stab}_{G}(c)$ and $h$ in $G$, where

$$
\left\|\delta_{i} \psi_{i}\right\|=\sup _{\substack{g, h \in G \\ c \in \mathcal{C}_{i}}}\left|\delta_{i} \psi_{i}(g, h)[c]\right| .
$$

Proof. (i) For every $g, h \in G$ we have

$$
\left|\delta_{i} \psi_{i}(g, h)[c]\right|=\left|\psi_{i}(h)[c g]-\psi_{i}(g h)[c]+\psi_{i}(g)[c]\right| \leq\left\|\delta_{i} \psi_{i}\right\| .
$$

Now if $g \in \operatorname{Stab}_{G}(c)$, then $\left|\psi_{i}(h)[c]-\psi_{i}(g h)[c]+\psi_{i}(g)[c]\right| \leq\left\|\delta_{i} \psi_{i}\right\|$, so

$$
\begin{gathered}
\left|\psi_{i}(g)[c]-\psi_{i}\left(g^{2}\right)[c]+\psi_{i}(g)[c]\right| \leq\left\|\delta_{i} \psi_{i}\right\|, \\
\left|\psi_{i}(h)[c]-\psi_{i}\left(g^{2} h\right)[c]+\psi_{i}\left(g^{2}\right)[c]\right| \leq\left\|\delta_{i} \psi_{i}\right\| .
\end{gathered}
$$

Using the triangle inequality we obtain

$$
\left|\psi_{i}(h)[c]-\psi_{i}\left(g^{2} h\right)[c]+2 \psi_{i}(g)[c]\right| \leq 2\left\|\delta_{i} \psi_{i}\right\| .
$$

Thus for any $n>0$ by induction we get

$$
\left|\psi_{i}(h)[c]-\psi_{i}\left(g^{n} h\right)[c]+n \psi_{i}(g)[c]\right| \leq n\left\|\delta_{i} \psi_{i}\right\|
$$

or

$$
\left|\frac{1}{n} \psi_{i}(h)[c]-\frac{1}{n} \psi_{i}\left(g^{n} h\right)[c]+\psi_{i}(g)[c]\right| \leq\left\|\delta_{i} \psi_{i}\right\| .
$$

Thus $\left|\psi_{i}(g)[c]\right| \leq\left\|\delta_{i} \psi_{i}\right\|$ for every $g \in \operatorname{Stab}_{G}(c)$.

(ii) Apply (i) and the triangle inequality.

Remark 3.3. Fix $c \in \mathcal{C}_{i}$ and fix a transversal $T$ for $\operatorname{Stab}_{G}(c)$ in $G$, so by Lemma 3.1, $\mathcal{C}_{i}=\{c t\}_{t \in T}$. For $t_{1} \in T$ let us consider the transversal $t_{1}^{-1} T$ for $\operatorname{Stab}_{G}\left(c t_{1}\right)$ in $G$. For every $h \in G$ since $G=\operatorname{Stab}_{G}\left(c t_{1}\right)\left(t_{1}^{-1} T\right)$, there exist unique elements $t_{2} \in T, k \in \operatorname{Stab}_{G}(c)$ such that $h=\left(t_{1}^{-1} k t_{1}\right) t_{1}^{-1} t_{2}$, where $t_{1}^{-1} k t_{1} \in \operatorname{Stab}_{G}\left(c t_{1}\right)$ and $t_{1}^{-1} t_{2} \in t_{1}^{-1} T$. By Lemma 3.2(ii), since $t_{1}^{-1} k t_{1} \in \operatorname{Stab}_{G}\left(c t_{1}\right)$, we have

$$
\left|\psi_{i}(h)\left[c t_{1}\right]-\psi_{i}\left(t_{1}^{-1} t_{2}\right)\left[c t_{1}\right]\right| \leq 2\left\|\delta_{i} \psi_{i}\right\| .
$$

Theorem 3.4. Let $G$ be a discrete group. Then $\mathcal{H}^{2}\left(\ell^{1}(G), \ell^{\infty}\left(\mathcal{C}_{i}\right)\right)$ is a Banach space, for every orbit $\mathcal{C}_{i}$ of $G$ in $S$. 
Proof. We wish to show that there exists a constant $M$ such that for every element of $\operatorname{Im} \delta_{i}$ like $\delta_{i} \psi_{i}$ there exists a $\bar{\psi}_{i} \in \mathcal{L}^{1}\left(\ell^{1}(G), \ell^{\infty}\left(\mathcal{C}_{i}\right)\right)$ such that $\delta_{i} \psi_{i}=\delta_{i} \bar{\psi}_{i}$ and $\left\|\bar{\psi}_{i}\right\| \leq M\left\|\delta_{i} \psi_{i}\right\|$.

Let $\psi_{i} \in \mathcal{L}^{1}\left(\ell^{1}(G), \ell^{\infty}\left(\mathcal{C}_{i}\right)\right)$. We fix $c \in \mathcal{C}_{i}$ and fix a transversal $T$ for $\operatorname{Stab}_{G}(c)$ in $G$, so by Lemma $3.1 \mathcal{C}_{i}=\{c t\}_{t \in T}$. For every $t_{1} \in T$ and every $g \in G$ we can choose unique elements $t_{2} \in T, k \in \operatorname{Stab}_{G}(c)$ such that $g=\left(t_{1}^{-1} k t_{1}\right) t_{1}^{-1} t_{2}$. Now for every $t_{1} \in T$ we define

$$
\bar{\psi}_{i}(g)\left[c t_{1}\right]=\psi_{i}(g)\left[c t_{1}\right]+\psi_{i}\left(t_{1}\right)[c]-\psi_{i}\left(t_{2}\right)[c] .
$$

By the boundary map for every $t_{0} \in T$ we have

$$
\delta_{i} \bar{\psi}_{i}(g, h)\left[c t_{0}\right]=\bar{\psi}_{i}(h)\left[c t_{0} g\right]-\bar{\psi}_{i}(g h)\left[c t_{0}\right]+\bar{\psi}_{i}(g)\left[c t_{0}\right] .
$$

Next we calculate all terms on the right-hand side of the above identity:

$$
\bar{\psi}_{i}(h)\left[c t_{0} g\right]=\bar{\psi}_{i}(h)\left[c t_{1}\right]=\psi_{i}(h)\left[c t_{1}\right]+\psi_{i}\left(t_{1}\right)[c]-\psi_{i}\left(t_{2}\right)[c],
$$

where $t_{1}$ is a unique element of $T$ such that $c t_{0} g=c t_{1}$ and $h=\left(t_{1}^{-1} k t_{1}\right) t_{1}^{-1} t_{2}$ for unique elements $t_{2} \in T, k \in \operatorname{Stab}_{G}(c)$.

Since $h=t_{1}^{-1} k t_{2}$, then $g h=t_{0}^{-1}\left(t_{0} g t_{1}^{-1} k\right) t_{2}$, but since $c t_{0} g t_{1}^{-1}=c$, then $t_{0} g t_{1}^{-1} \in$ $\operatorname{Stab}_{G}(c)$, so $t_{0} g t_{1}^{-1} k \in \operatorname{Stab}_{G}(c)$ and so

$$
\bar{\psi}_{i}(g h)\left[c t_{0}\right]=\psi_{i}(g h)\left[c t_{0}\right]+\psi_{i}\left(t_{0}\right)[c]-\psi_{i}\left(t_{2}\right)[c] .
$$

Finally since $g=t_{0}^{-1}\left(t_{0} g t_{1}^{-1}\right) t_{1}$, then

$$
\bar{\psi}_{i}(g)\left[c t_{0}\right]=\psi_{i}(g)\left[c t_{0}\right]+\psi_{i}\left(t_{0}\right)[c]-\psi_{i}\left(t_{1}\right)[c] .
$$

Thus substituting (3.3), (3.4) and (3.5) in equation (3.2) we obtain

$$
\delta_{i} \bar{\psi}_{i}(g, h)\left[c t_{0}\right]=\delta_{i} \psi_{i}(g, h)\left[c t_{0}\right]
$$

By inequality (3.1) we have

$$
\begin{aligned}
\left|\bar{\psi}_{i}(g)\left[c t_{1}\right]\right| & =\left|\psi_{i}(g)\left[c t_{1}\right]+\psi_{i}\left(t_{1}\right)[c]-\psi_{i}\left(t_{2}\right)[c]\right| \\
& \leq\left|\psi_{i}(g)\left[c t_{1}\right]-\psi_{i}\left(t_{1}^{-1} t_{2}\right)\left[c t_{1}\right]\right|+\left|\psi_{i}\left(t_{1}^{-1} t_{2}\right)\left[c t_{1}\right]+\psi_{i}\left(t_{1}\right)[c]-\psi_{i}\left(t_{2}\right)[c]\right| \\
& \leq 2\left\|\delta_{i} \psi_{i}\right\|+\left\|\delta_{i} \psi_{i}\right\| \\
& \leq 3\left\|\delta_{i} \psi_{i}\right\| .
\end{aligned}
$$

Therefore $\left|\bar{\psi}_{i}(g)\left[c t_{1}\right]\right| \leq 3\left\|\delta_{i} \psi_{i}\right\|$ for every $g \in G$ and every $t_{1} \in T$, and so we have $\left\|\bar{\psi}_{i}\right\| \leq 3\left\|\delta_{i} \psi_{i}\right\|$ for every $i \in I$.

Let us consider the $\ell^{\infty}$-direct sum $\bigoplus_{i \in I}^{\infty} \mathcal{L}^{n}\left(\ell^{1}(G), \ell^{\infty}\left(\mathcal{C}_{i}\right)\right)$ which is the subset of the Cartesian product $\prod_{i \in I} \mathcal{L}^{n}\left(\ell^{1}(G), \ell^{\infty}\left(\mathcal{C}_{i}\right)\right)$ consisting of all $\left\{\phi_{i}\right\}_{i \in I}$, where $\phi_{i} \in \mathcal{L}^{n}\left(\ell^{1}(G), \ell^{\infty}\left(\mathcal{C}_{i}\right)\right)$ and $\left\|\left\{\phi_{i}\right\}\right\|=\sup _{i \in I}\left\|\phi_{i}\right\|<\infty$. The boundary map

$$
\bigoplus_{i \in I} \delta_{i}^{n}: \bigoplus_{i \in I}^{\infty} \mathcal{L}^{n-1}\left(\ell^{1}(G), \ell^{\infty}\left(\mathcal{C}_{i}\right)\right) \rightarrow \bigoplus_{i \in I}^{\infty} \mathcal{L}^{n}\left(\ell^{1}(G), \ell^{\infty}\left(\mathcal{C}_{i}\right)\right)
$$

is defined by

$$
\bigoplus_{i \in I} \delta_{i}^{n}\left(\left\{\phi_{i}\right\}_{i \in I}\right)=\left\{\delta_{i}^{n} \phi_{i}\right\}_{i \in I}
$$

Lemma 3.5. There exists an isometric isomorphism from $\mathcal{L}^{n}\left(\ell^{1}(G), \ell^{\infty}(S)\right)$ onto $\bigoplus_{i \in I}^{\infty} \mathcal{L}^{n}\left(\ell^{1}(G), \ell^{\infty}\left(\mathcal{C}_{i}\right)\right)$. 
Proof. Let $\phi \in \mathcal{L}^{n}\left(\ell^{1}(G), \ell^{\infty}(S)\right)$. Then for every $i \in I$ and $c \in \mathcal{C}_{i}$ we define $\phi_{i}$ by

$$
\phi_{i}\left(g_{1}, \ldots, g_{n}\right)[c]=\phi\left(g_{1}, \ldots, g_{n}\right)(c) \text {. }
$$

Now define

$$
T_{n}: \mathcal{L}^{n}\left(\ell^{1}(G), \ell^{\infty}(S)\right) \rightarrow \bigoplus_{i \in I}^{\infty} \mathcal{L}^{n}\left(\ell^{1}(G), \ell^{\infty}\left(\mathcal{C}_{i}\right)\right)
$$

by $T_{n}(\phi)=\left\{\phi_{i}\right\}_{i \in I}$.

(i) $T_{n}$ is injective; if $T_{n}\left(\phi_{1}\right)=T_{n}\left(\phi_{2}\right)$, then by the definition of $T_{n}$ we have $\phi_{1_{i}}=\phi_{2_{i}}$ for every $i \in I$, which implies that $\phi_{1}=\phi_{2}$ on $G \times \cdots \times G \times \mathcal{C}_{i}$ for every $i \in I$, but $S=\bigcup_{i \in I} \mathcal{C}_{i}$ and $\mathcal{C}_{i} \cap \mathcal{C}_{j}=\emptyset$ if $i \neq j$, so $\phi_{1}=\phi_{2}$ on $G \times \cdots \times G \times S$.

(ii) $T_{n}$ is surjective; let $\left\{\phi_{i}\right\}_{i \in I}$ be an element of $\bigoplus_{i \in I}^{\infty} \mathcal{L}^{n}\left(\ell^{1}(G), \ell^{\infty}\left(\mathcal{C}_{i}\right)\right)$. For every $x \in S$ there exists a unique $i \in I$ such that $x \in \mathcal{C}_{i}$. Now we define $\phi: \ell^{1}(G) \times$ $\cdots \times \ell^{1}(G) \rightarrow \ell^{\infty}(S)$ by $\phi\left(g_{1}, \ldots, g_{n}\right)(x)=\phi_{i}\left(g_{1}, \ldots, g_{n}\right)[x]$, since $\sup _{i \in I}\left\|\phi_{i}\right\|<\infty$; then $\phi \in \mathcal{L}^{n}\left(\ell^{1}(G), \ell^{\infty}\left(\mathcal{C}_{i}\right)\right)$ and $T_{n}(\phi)=\left\{\phi_{i}\right\}_{i \in I}$.

Finally

$$
\begin{aligned}
\left\|T_{n} \phi\right\| & =\left\|\left\{\phi_{i}\right\}_{i \in I}\right\| \\
& =\sup _{i \in I}\left\{\left\|\phi_{i}\right\|\right\}=\|\phi\| .
\end{aligned}
$$

Hence $T_{n}$ is isometric.

Lemma 3.6. $\bigoplus_{i \in I} \delta_{i}^{n} \circ T_{n-1}=T_{n} \circ \delta^{n}$.

Proof. Let $\phi \in \mathcal{L}^{n}\left(\ell^{1}(G), \ell^{\infty}(S)\right)$. Then

$$
\begin{aligned}
\bigoplus_{i \in I} \delta_{i}^{n} \circ T_{n-1}(\phi) & =\bigoplus_{i \in I} \delta_{i}^{n}\left(T_{n-1}(\phi)\right) \\
& =\bigoplus_{i \in I} \delta_{i}^{n}\left(\left\{\phi_{i}\right\}_{i \in I}\right)=\left\{\delta_{i}^{n} \phi_{i}\right\}_{i \in I} .
\end{aligned}
$$

On the other hand

$$
T_{n} \circ \delta^{n}(\phi)=T_{n}\left(\delta^{n} \phi\right)=\left\{\delta_{i}^{n} \phi_{i}\right\}_{i \in I} .
$$

Corollary 3.7. An isomorphism of vector spaces

$$
\mathcal{H}^{n}\left(\ell^{1}(G), \ell^{\infty}(S)\right) \cong \bigoplus_{i \in I}^{\infty} \mathcal{H}^{n}\left(\ell^{1}(G), \ell^{\infty}\left(\mathcal{C}_{i}\right)\right)
$$

holds.

\section{QuASIDERIVATIONS AND PSEUdOdERIVATIONS}

Let $G$ be a discrete group, and let $S$ be a $G$-set with trivial action on the lefthand side. The inclusion homomorphism $C_{b}^{n}\left(G, \ell^{\infty}(S)\right) \rightarrow C^{n}\left(G, \ell^{\infty}(S)\right)$ induces a homomorphism $\zeta: \mathcal{H}_{b}^{n}\left(G, \ell^{\infty}(S)\right) \rightarrow H^{n}\left(G, \ell^{\infty}(S)\right)$, which in general is neither injective nor surjective. The image of this homomorphism is called the bounded part of $H^{n}\left(G, \ell^{\infty}(S)\right)$ and will be denoted by $\mathcal{H}_{b, 1}^{n}\left(G, \ell^{\infty}(S)\right)$. Note that

$$
\mathcal{H}_{b, 1}^{n}\left(G, \ell^{\infty}(S)\right)=\frac{\operatorname{ker} \delta_{b}^{n+1}}{\operatorname{Im} \delta^{n} \cap \operatorname{ker} \delta_{b}^{n+1}} .
$$


The seminorm on $\mathcal{H}_{b}^{n}(G, X)$ induces a seminorm on the bounded part of $H^{n}(G, X)$ by

$$
\|[f]\|_{\mathcal{H}_{b, 1}^{n}}=\inf \left\{\|[f+g]\|_{\mathcal{H}_{b}^{n}}: g \in \operatorname{Im} \delta^{n} \cap \operatorname{ker} \delta_{b}^{n+1}\right\} .
$$

Proposition 4.1. The space $\mathcal{H}_{b, 1}^{n}\left(G, \ell^{\infty}(S)\right)$ with the induced seminorm is a $B a$ nach space.

Proof. Let $f \in C_{b}^{n}\left(G, \ell^{\infty}(S)\right)$. We denote by $[f]$ the class of the element $f$ in $\mathcal{H}_{b, 1}^{n}\left(G, \ell^{\infty}(S)\right)$. Then

$$
\|[f]\|_{\mathcal{H}_{b, 1}^{n}}=\inf \left\{\|[f+g]\|_{\mathcal{H}_{b}^{n}}: g \in \operatorname{Im} \delta^{n} \cap \operatorname{ker} \delta_{b}^{n+1}\right\} .
$$

But

$$
\|[f+g]\|_{\mathcal{H}_{b}^{n}}=\inf \left\{\|f+g+h\|_{\infty}: h \in \operatorname{Im} \delta_{b}^{n}\right\} .
$$

Since $[f]$ is a nonzero element of $\mathcal{H}^{n}\left(G, \ell^{\infty}(S)\right) \cong \mathcal{H}_{n}\left(G, \ell^{1}(S)\right)^{*}$, then there is $[x] \in$ $\mathcal{H}_{n}\left(G, \ell^{1}(S)\right)$ such that $f(x) \neq 0$. But since $g \in \operatorname{Im} \delta^{n} \cap \operatorname{ker} \delta_{b}^{n+1}$ and $h \in \operatorname{Im} \delta_{b}^{n}$, then there exist $g_{1} \in C^{n-1}\left(G, \ell^{\infty}(S)\right)$ and $h_{1} \in C_{b}^{n-1}\left(G, \ell^{\infty}(S)\right)$ such that $g=\delta^{n} g_{1}$ and $h=\delta_{b}^{n} h_{1}$. Since $x \in \operatorname{ker} d_{n}$ we have

$(f+g+h)(x)=f(x)+\delta^{n} g_{1}(x)+\delta_{b}^{n} h_{1}(x)=f(x)+g_{1}\left(d_{n}(x)\right)+h_{1}\left(d_{n}(x)\right)=f(x)$. Then by (4.1) and (4.2) $\|f\|_{\mathcal{H}_{b, 1}}^{n} \geq|f(x)|>0$. So we proved that if $[f] \neq 0$ in $\mathcal{H}_{b, 1}^{n}\left(G, \ell^{\infty}(S)\right)$, then $\|f\|_{\mathcal{H}_{b, 1}}^{n} \neq 0$.

Now we define $\mathcal{H}_{b, 2}^{n}\left(G, \ell^{\infty}(S)\right) \subseteq \mathcal{H}_{b}^{n}\left(G, \ell^{\infty}(S)\right)$ to be the subspace

$$
\mathcal{H}_{b, 2}^{n}\left(G, \ell^{\infty}(S)\right)=\frac{\operatorname{Im} \delta^{n} \cap \operatorname{ker} \delta_{b}^{n+1}}{\operatorname{Im} \delta_{b}^{n}} .
$$

Proposition 4.2. A natural isomorphism of vector spaces with the seminorms

$$
\mathcal{H}_{b, 1}^{n}\left(G, \ell^{\infty}(S)\right) \cong \frac{\mathcal{H}_{b}^{n}\left(G, \ell^{\infty}(S)\right)}{\mathcal{H}_{b, 2}^{n}\left(G, \ell^{\infty}(S)\right)}
$$

holds.

Proof. The following exact sequence exists:

$$
0 \rightarrow \frac{\operatorname{Im} \delta^{n} \cap \operatorname{ker} \delta_{b}^{n+1}}{\operatorname{Im} \delta_{b}^{n}} \rightarrow \frac{\operatorname{ker} \delta_{b}^{n+1}}{\operatorname{Im} \delta_{b}^{n}} \rightarrow \frac{\operatorname{ker} \delta_{b}^{n+1}}{\operatorname{Im} \delta^{n} \cap \operatorname{ker} \delta_{b}^{n+1}} \rightarrow 0 .
$$

So by definition we have the result. The seminorm is defined as a seminorm on a quotient space.

Corollary 4.3. An isomorphism of vector spaces

$$
\mathcal{H}_{b}^{n}\left(G, \ell^{\infty}(S)\right) \cong \mathcal{H}_{b, 1}^{n}\left(G, \ell^{\infty}(S)\right) \oplus \mathcal{H}_{b, 2}^{n}\left(G, \ell^{\infty}(S)\right)
$$

holds.

Throughout we drop the subscript $i$ on $\delta_{i}$ and the elements of $\mathcal{L}^{1}\left(\ell^{1}(G), \ell^{\infty}\left(\mathcal{C}_{i}\right)\right)$.

Definition 4.4. A function $\psi \in C^{n}\left(G, \ell^{\infty}(S)\right.$ is called a quasiderivation if there is a constant $C \geq 0$ such that for every $g, h \in G$ and $c \in \mathcal{C}_{i}$

$$
|\psi(h)(c g)-\psi(g h)(c)+\psi(g)(c)| \leq C,
$$

we denote the space of quasiderivations by $Q D\left(G, \ell^{\infty}(S)\right)$. Note that a function $\psi \in Q D\left(G, \ell^{\infty}(S)\right)$ if and only if $\delta^{1}(\psi)$ is bounded. 
Definition 4.5. A function $\psi \in Q D\left(G, \ell^{\infty}(S)\right)$ is called a pseudoderivation if $\psi\left(g^{n}\right)(c)=n \psi(g)(c)$ for every $g \in \operatorname{Stab}_{G}(c), c \in S$ and $n \in \mathbb{Z}$. We denote by $P D\left(G, \ell^{\infty}(S)\right)$ the space of pseudoderivations, and by $D\left(G, \ell^{\infty}(S)\right)$ the space of all derivations from $G$ to $\ell^{\infty}(S)$.

Proposition 4.6. If $c \in \mathcal{C}_{i}$ is some fixed element, then a vector spaces isomorphism

$$
\mathcal{H}_{b, 2}^{2}\left(\operatorname{Stab}_{G}(c), \ell^{\infty}\left(\mathcal{C}_{i}\right)\right) \cong \frac{Q D\left(\operatorname{Stab}_{G}(c), \ell^{\infty}\left(\mathcal{C}_{i}\right)\right)}{D\left(\operatorname{Stab}_{G}(c), \ell^{\infty}\left(\mathcal{C}_{i}\right)\right) \oplus C_{b}^{1}\left(\operatorname{Stab}_{G}(c), \ell^{\infty}\left(\mathcal{C}_{i}\right)\right)}
$$

holds.

Proof. We abbreviate $\operatorname{Stab}_{G}(c)$ by $H$. To begin note that if $\psi \in D\left(H, \ell^{\infty}\left(\mathcal{C}_{i}\right)\right)$ for every $g, h \in H$ we have $\psi(g h)[c]=\psi(h)[c]+\psi(g)[c]$. So by induction for every $n \in \mathbb{N}$ we have $\psi\left(g^{n}\right)[c]=n \psi(g)[c]$; now if $\psi \in C_{b}^{1}\left(H, \ell^{\infty}\left(\mathcal{C}_{i}\right)\right)$, then $\psi(g)[c]=0$, that is, $D\left(H, \ell^{\infty}\left(\mathcal{C}_{i}\right)\right) \cap C_{b}^{1}\left(H, \ell^{\infty}\left(\mathcal{C}_{i}\right)\right)=\{0\}$. We have a sequence

$$
Q D\left(H, \mathcal{C}_{i}\right) \stackrel{\delta^{1}}{\longrightarrow} \operatorname{Im} \delta^{1} \cap \operatorname{ker} \delta_{b}^{2} \stackrel{\pi}{\longrightarrow} \frac{\operatorname{Im} \delta^{1} \cap \operatorname{ker} \delta_{b}^{2}}{\operatorname{Im} \delta_{b}^{1}},
$$

where $\delta^{1}$ is onto and $\pi$ is the canonical mapping. For every $\psi \in Q D\left(H, \mathcal{C}_{i}\right)$

$$
\begin{aligned}
\pi\left(\delta^{1}(\psi)=0\right. & \Longleftrightarrow \delta^{1} \psi \in \operatorname{Im} \delta_{b}^{1} \\
& \Longleftrightarrow \delta^{1}(\psi)=\delta_{b}^{1}(g) \quad \text { for some } g \in C_{b}^{1}\left(H, \ell^{\infty}\left(\mathcal{C}_{i}\right)\right) \\
& \Longleftrightarrow \delta^{1}(\psi-g)=0 \Longleftrightarrow \psi-g \in D\left(H, \mathcal{C}_{i}\right) .
\end{aligned}
$$

So $\psi=(\psi-g)+g \in D\left(H, \ell^{\infty}\left(\mathcal{C}_{i}\right)\right) \oplus C_{b}^{1}\left(H, \ell^{\infty}\left(\mathcal{C}_{i}\right)\right)$, that is, we have proved that $\operatorname{ker} \pi \circ \delta^{1}=D\left(H, \ell^{\infty}\left(\mathcal{C}_{i}\right)\right) \oplus C_{b}^{1}\left(H, \ell^{\infty}\left(\mathcal{C}_{i}\right)\right)$, and this finishes the proof.

Proposition 4.7. Let $\psi$ be a real-valued submultiplicative map on a Banach algebra $X$. Then $\lim _{n \rightarrow \infty} \psi\left(x^{n}\right)^{1 / n}$ exists.

Proof. Set $r=\inf _{n \geq 1} \psi\left(x^{n}\right)^{1 / n}$. We will show that $\varlimsup_{n} \psi\left(x^{n}\right)^{1 / n} \leq r$. For any $\epsilon>0$, choose $m$ such that $\psi\left(x^{m}\right)^{1 / m} \leq r+\epsilon$. For arbitrary $n$, write $n=p m+q$ where $0 \leq q \leq m-1$. Then

$$
\psi\left(x^{n}\right)^{1 / n} \leq \psi\left(x^{m}\right)^{p / n}+\psi(x)^{q / n} \leq(r+\epsilon)^{p m / n}+\psi(x)^{q / n} .
$$

Since $p m / n \rightarrow 1$ and $q / n \rightarrow 0$ as $n \rightarrow \infty$, we have $\psi\left(x^{n}\right)^{1 / n} \leq r+\epsilon$. Since $\epsilon$ was arbitrary, we have $\varlimsup_{n} \psi\left(x^{n}\right)^{1 / n} \leq r$.

Corollary 4.8. If $\psi \in Q D\left(G, \ell^{\infty}(S)\right)$, then $\lim _{n \rightarrow \infty} \psi\left(g^{n}\right)(c) / n$ exists for every $g \in \operatorname{Stab}_{G}(c)$.

Proof. Decomposing $\psi$ into its real and imaginary parts, we can reduce the problem to the case in which $\psi$ is real-valued. Since $\psi \in Q D\left(G, \ell^{\infty}(S)\right)$, then for every $g \in \operatorname{Stab}_{G}(c)$ and $h \in G$ we have

$$
-C \leq \psi(h)(c)-\psi(g h)(c)+\psi(g)(c) \leq C
$$

and so

$$
e^{(\psi(g h)(c)+C)} \leq e^{(\psi(g)(c)+C)} e^{(\psi(h)(c)+C)} ;
$$

then by Proposition $4.7 \lim _{n \rightarrow \infty}\left(e^{\psi\left(g^{n}\right)(c)+C}\right)^{1 / n}$ exists. Therefore

$$
\lim _{n \rightarrow \infty} \frac{\left(\psi\left(g^{n}\right)(c)+C\right)}{n}=\lim _{n \rightarrow \infty} \frac{\psi\left(g^{n}\right)(c)}{n}
$$

exists. 
Remark 4.9. Let $\psi \in Q D\left(G, \ell^{\infty}\left(\mathcal{C}_{i}\right)\right)$. For every $g \in \operatorname{Stab}_{G}(c)$ we define $\tilde{\psi}$ : $\ell^{\mathbf{1}}\left(\operatorname{Stab}_{G}(c)\right) \rightarrow \ell^{\infty}\left(\mathcal{C}_{i}\right)$ by

$$
\tilde{\psi}(g)[c]=\lim _{n \rightarrow \infty} \frac{\psi\left(g^{n}\right)[c]}{n} .
$$

Note that for every $g \in \operatorname{Stab}_{G}(c)$

$$
\tilde{\psi}\left(g^{k}\right)[c]=k \lim _{n \rightarrow \infty} \frac{\psi\left(g^{k n}\right)[c]}{k n}=k \tilde{\psi}(g)[c] .
$$

Since for every $g \in \operatorname{Stab}_{G}(c)$

$$
\sum_{n=0}^{\infty} \frac{\delta \psi\left(g^{2^{n}}, g^{2^{n}}\right)[c]}{2^{n+1}}=\psi(g)[c]-\frac{\psi\left(g^{2^{N}}\right)[c]}{2^{N}}+\sum_{n=N}^{\infty} \frac{\delta \psi\left(g^{2^{n}}, g^{2^{n}}\right)[c]}{2^{n+1}},
$$

so $\psi(g)[c]-\tilde{\psi}(g)[c]=\sum_{n=0}^{\infty} \frac{\delta \psi\left(g^{2^{n}}, g^{2^{n}}\right)[c]}{2^{n+1}}$. But since $\psi \in Q D\left(G, \ell^{\infty}\left(\mathcal{C}_{i}\right)\right)$ we have $\left|\delta \psi\left(g^{2^{n}}, g^{2^{n}}\right)[c]\right|<C$. Then

$$
\psi(g)[c] \approx \tilde{\psi}(g)[c],
$$

where the notation $\approx$ means $|\psi(g)[c]-\tilde{\psi}(g)[c]|$ is bounded for every $g \in G$ and $c \in \mathcal{C}_{i}$.

Next by Remark 3.3 for every $h \in G$ and $t_{1} \in T$ there exist unique elements $t_{2} \in T, g \in \operatorname{Stab}_{G}(c)$ such that $h=t_{1}^{-1} g t_{2}$ and since $\psi \in Q D\left(G, \ell^{\infty}(S)\right)$ we have

$$
\begin{aligned}
\psi\left(t_{1}^{-1} g t_{2}\right)\left[c t_{1}\right] & \approx \psi\left(g t_{2}\right)[c]+\psi\left(t_{1}^{-1}\right)\left[c t_{1}\right] \\
& \approx \psi\left(t_{2}\right)[c]+\psi(g)[c]+\psi\left(t_{1}^{-1}\right)\left[c t_{1}\right] .
\end{aligned}
$$

But from

$$
\begin{aligned}
\psi(e)\left[c t_{1}\right]=\psi\left(t_{1}^{-1} t_{1}\right)\left[c t_{1}\right] & \approx \psi\left(t_{1}\right)[c]+\psi\left(t_{1}^{-1}\right)\left[c t_{1}\right] \\
& \approx \psi(e)\left[c t_{1}\right]+\psi(e)\left[c t_{1}\right]
\end{aligned}
$$

we have $\psi(e)\left[c t_{1}\right] \approx 0$ and so $\psi\left(t_{1}^{-1}\right)\left[c t_{1}\right] \approx-\psi\left(t_{1}\right)[c]$. Hence by (4.7)

$$
\psi\left(t_{1}^{-1} g t_{2}\right)\left[c t_{1}\right] \approx \psi(g)[c]+\psi\left(t_{2}\right)[c]-\psi\left(t_{1}\right)[c] .
$$

Using (4.6) and (4.8) we obtain

$$
\psi(h)\left[c t_{1}\right]=\psi\left(t_{1}^{-1} g t_{2}\right)\left[c t_{1}\right] \approx \tilde{\psi}(g)[c]+\psi\left(t_{2}\right)[c]-\psi\left(t_{1}\right)[c] .
$$

Now let us define $\bar{\psi}: \ell^{1}(G) \rightarrow \ell^{\infty}\left(\mathcal{C}_{i}\right)$

$$
\bar{\psi}(h)\left[c t_{1}\right]=\tilde{\psi}(g)[c]+\psi\left(t_{2}\right)[c]-\psi\left(t_{1}\right)[c] .
$$

Then by (4.9) $\psi(h)\left[c t_{1}\right] \approx \bar{\psi}(h)\left[c t_{1}\right]$ for every $h \in G, t_{1} \in T$, that is, $\psi-\bar{\psi} \in$ $C_{b}^{1}\left(G, \ell^{\infty}\left(\mathcal{C}_{i}\right)\right)$. We claim that $\bar{\psi} \in P D\left(G, \ell^{\infty}(S)\right)$. To see this we have to show for every $h \in \operatorname{Stab}_{G}\left(c t_{0}\right)$ and $t_{0} \in T$

$$
\bar{\psi}\left(h^{k}\right)\left[c t_{0}\right]=k \bar{\psi}(h)\left[c t_{0}\right] .
$$

Since $h \in \operatorname{Stab}_{G}\left(c t_{0}\right)$ we have $t_{0} h t_{0}^{-1} \in \operatorname{Stab}_{G}(c)$ and so $h^{k}=t_{0}^{-1}\left(t_{0} h^{k} t_{0}^{-1}\right) t_{0}$. Therefore by (4.4) and the definition of $\bar{\psi}$ we have

$$
\begin{aligned}
\bar{\psi}\left(h^{k}\right)\left[c t_{0}\right] & =\tilde{\psi}\left(t_{0} h^{k} t_{0}^{-1}\right)[c]+\psi\left(t_{0}\right)[c]-\psi\left(t_{0}\right)[c] \\
& =k \tilde{\psi}\left(t_{0} h t_{0}^{-1}\right)[c]=k \bar{\psi}(h)\left[c t_{0}\right] .
\end{aligned}
$$


Theorem 4.10. If $c \in \mathcal{C}_{i}$ is some fixed element, then a vector spaces isomorphism

$$
\frac{Q D\left(\operatorname{Stab}_{G}(c), \ell^{\infty}\left(\mathcal{C}_{i}\right)\right)}{D\left(\operatorname{Stab}_{G}(c), \ell^{\infty}\left(\mathcal{C}_{i}\right)\right) \oplus C_{b}^{1}\left(\operatorname{Stab}_{G}(c), \ell^{\infty}\left(\mathcal{C}_{i}\right)\right)} \cong \frac{P D\left(\operatorname{Stab}_{G}(c), \ell^{\infty}\left(\mathcal{C}_{i}\right)\right)}{D\left(\operatorname{Stab}_{G}(c), \ell^{\infty}\left(\mathcal{C}_{i}\right)\right)}
$$

holds.

Proof. Again we abbreviate $\operatorname{Stab}_{G}(c)$ by $H$. For every $\psi \in Q D\left(H, \ell^{\infty}\left(\mathcal{C}_{i}\right)\right)$ Remark 4.9 gives $\bar{\psi}$ in $P D\left(H, \ell^{\infty}\left(\mathcal{C}_{i}\right)\right)$ such that $\psi-\bar{\psi} \in C_{b}^{1}\left(H, \ell^{\infty}\left(\mathcal{C}_{i}\right)\right)$. Now we define

$$
\xi: Q D\left(H, \ell^{\infty}\left(\mathcal{C}_{i}\right)\right) \longrightarrow \frac{P D\left(H, \ell^{\infty}\left(\mathcal{C}_{i}\right)\right)}{D\left(H, \ell^{\infty}\left(\mathcal{C}_{i}\right)\right)}
$$

by $\xi(\psi)=\bar{\psi}+D\left(H, \ell^{\infty}\left(\mathcal{C}_{i}\right)\right)$. Note that $\psi \in \operatorname{ker} \xi$ if and only if $\bar{\psi}+D\left(H, \ell^{\infty}\left(\mathcal{C}_{i}\right)\right)=$ $D\left(H, \ell^{\infty}\left(\mathcal{C}_{i}\right)\right)$ if and only if $\bar{\psi} \in D\left(H, \ell^{\infty}\left(\mathcal{C}_{i}\right)\right)$. Therefore $\psi=(\psi-\bar{\psi})+\psi \in$ $D\left(H, \ell^{\infty}\left(\mathcal{C}_{i}\right)\right) \oplus C_{b}^{1}\left(H, \ell^{\infty}\left(\mathcal{C}_{i}\right)\right)$, that is, $\operatorname{ker} \xi=D\left(H, \ell^{\infty}\left(\mathcal{C}_{i}\right)\right) \oplus C_{b}^{1}\left(H, \ell^{\infty}\left(\mathcal{C}_{i}\right)\right)$.

The following theorem is a consequence of Proposition 4.6 and Theorem 4.10 .

Theorem 4.11. If $c \in \mathcal{C}_{i}$ is some fixed element, then an isomorphism of vector spaces

$$
\mathcal{H}_{b, 2}^{2}\left(\operatorname{Stab}_{G}(c), \ell^{\infty}\left(\mathcal{C}_{i}\right)\right) \cong \frac{P D\left(\operatorname{Stab}_{G}(c), \ell^{\infty}\left(\mathcal{C}_{i}\right)\right)}{D\left(\operatorname{Stab}_{G}(c), \ell^{\infty}\left(\mathcal{C}_{i}\right)\right)}
$$

holds.

Remark 4.12. One should note that the result of Grønbæk [3, Section 6] has shown that

$$
\mathcal{H}^{2}\left(\ell^{1}(G), \ell^{\infty}\left(\mathcal{C}_{i}\right)\right) \cong \mathcal{H}^{2}\left(\ell^{1}\left(\operatorname{Stab}_{G}(c)\right), \ell^{\infty}\left(\mathcal{C}_{i}\right)\right)
$$

where $c \in \mathcal{C}_{i}$ is some fixed element. The Grønbæk argument is a generalization of the method of Johnson [6, 2.7].

Finally we apply Remark 4.12 and Theorem 4.10 for the following result.

Theorem 4.13. If $c \in \mathcal{C}_{i}$ is some fixed element, then an isomorphism of vector spaces

$$
\mathcal{H}_{b, 2}^{2}\left(\ell^{1}(G), \ell^{\infty}\left(\mathcal{C}_{i}\right)\right) \cong \frac{P D\left(\operatorname{Stab}_{G}(c), \ell^{\infty}\left(\mathcal{C}_{i}\right)\right)}{D\left(\operatorname{Stab}_{G}(c), \ell^{\infty}\left(\mathcal{C}_{i}\right)\right)}
$$

holds.

\section{ACKNOWLEDGMENT}

The author wishes to thank the referee for bringing [9] to his attention.

\section{REFERENCES}

[1] R. Brooks, Some remarks on bounded cohomology, Riemann surfaces and related topics, Ann. of Math. Studies 97 (1981), 53-63. MR0624804 (83a:57038)

[2] R. I. Grigorchuk, Some results on bounded cohomology, London Math. Soc. Lecture Note series. 203 (1995), 111-163. MR1320279 (96j:20073)

[3] N. Grønbæk, Some concepts from group cohomology in the Banach algebra context. Proc. Banach Algebras 97 Conference, Blaubeuren (1998), pp. 205-222. MR1656607|(2000d:46087)

[4] A. Ya. Helemskii, The homology of Banach and topological algebras (Math. and its Applications), 1989. MR1093462(92d:46178)

[5] N. V. Ivanov, Second bounded cohomology group, J. Soviet Math. 167 (1988), 117-120. MR0964260(90a:55015)

[6] B. E. Johnson, Cohomology in Banach algebras, Memoirs Amer. Math. Soc. 127 (1972). MR0374934 (51:11130) 
[7] S. Matsumoto and S. Moruta, Bounded cohomology of certain groups of homeomorphisms, Proc. Amer. Math. Soc. 94 (1985), 539-544. MR0787909 (87e:55006)

[8] A. Pourabbas, Second cohomology group of group algebras with coefficients in iterated duals. Proc. Amer. Math. Soc. 132 (2004) no. 5, 1403-1410. MR2053346 (2005b:43003)

[9] T. Soma, Existence of non-Banach bounded cohomology, Topology 37 (1998), no. 1, 179-193. MR.1480885(99e:55010)

Faculty of Mathematics and Computer Science, Amirkabir University of Technology, 424 Hafez Avenue, Tehran 15914, Iran

E-mail address: arpabbas@aut.ac.ir 